\title{
ANTIMICROBIALS
}

\section{Review of Amphotericin B for Invasive Fungal Infections in Neonates and Children}

\author{
Jeeson C Unni
}

\begin{abstract}
Formulations of amphotericin B have come a long way since it was initially discovered. Studies of antifungals in the pediatric age-group are limited to those for cutaneous fungal infections. There are a few studies on the pharmacokinetics and safety of amphotericin B, and most dosage recommendations are extrapolated from adult data. A review of its use in the treatment of invasive fungal infections in neonates, children, and adolescents is the focus of this article.
\end{abstract}

Keywords: Amphotericin B, Antifungals, Children and adolescents, Invasive fungal infections, Neonate.

Pediatric Infectious Disease (2020): 10.5005/jp-journals-10081-1240

\section{INTRODUCTION}

Nystatin was the first antifungal used in humans. ${ }^{1}$ That was in the year 1950, 285 years after thrush was recognized as a fatal disease. Fungi being eukaryotic like humans and unlike the prokaryotic bacteria need to be treated with drugs that act on the organism without affecting the infected human cell. The process of product characterization, and optimal drug formulation to overcome this hurdle has resulted in great delay in the development of antifungals.

The treatment of critically ill preterm babies, term neonates, and children in NICUs and PICUs; need for invasive procedures in these settings; increased use of very broad-spectrum antibiotics; organ and bone marrow transplantations; immunosuppression due to HIV and childhood cancer; and survival of children with primary immunodeficiency has resulted in large number of invasive fungal infections (IFI) ${ }_{i}^{2-6}$ necessitated study of antifungals in children and amphotericin B, the oldest antifungal used for IFI in neonates and children, got its share. Most recommendations for dosing of antifungals in pediatrics are extrapolated from adult studies. However, the pathogenesis of fungal infections and pharmocokinetics, safety, and efficacy of antifungals are significantly different in neonates and children, and these differences need to be addressed when formulating treatment regimens in this population.

\section{Pharmacologic Characteristics of Antifungals in Neonates and Children}

Studies have demonstrated nonlinear relationship between size of the patient (weight of the neonate or child) and absolute value for clearance of an antifungal for a given weight. ${ }^{7}$ This means that drug exposure and hence dosing would change in a nonlinear way across a range of weights, and therefore recommendations for dosing in neonates and children should preferably not be based on adult data. Examples of pharmacokinetics of amphotericin B lipid complex, micafungin, and fluconazole highlight this phenomenon.

Furthermore, pharmacokinetics of drugs change with the maturity of hepatic clearance mechanisms and renal function which is especially rapid in neonates; they also have less adipose
IAP Drug Formulary, Kochi, Kerala, India; Department of Child Health and Adolescent Medicine, Aster Medcity, Kochi, Kerala, India

Corresponding Author: Jeeson C Unni, IAP Drug Formulary, Kochi, Kerala, India; Department of Child Health and Adolescent Medicine, Aster Medcity, Kochi, Kerala, India, Phone: +91 9847245207, e-mail: jeeson1955@gmail.com

How to cite this article: Unni JC. Review of Amphotericin B for Invasive Fungal Infections in Neonates and Children. Pediatr Inf Dis 2020;2(3):114-117.

Source of support: Nil

Conflict of interest: None

tissue and more total body water-all of which could affect dosing guidelines.

\section{Dosing Strategies}

A number of dosing strategies have been tried of which probably the most accurate is that which is based on surface area of the child; assessment of surface area of newborn child is difficult and often not accurate. The dosages of amphotericin B have been recommended on a "mg/ $\mathrm{kg}^{-1 "}$ basis (Table 1) which carries a significant risk of underdosing in neonates and children if the reference dose is the adult dosage.

\section{Mechanism of Action of Antifungals Used in IFI with Emphasis on Amphotericin B}

The antifungal drugs are classified into 3 groups —antifungal agents acting on plasmatic membranes (azoles, polyenes), drugs acting on synthesis of nucleic acids (5-flucytosine), or those acting on fungal cell walls (echinocandins). ${ }^{8}$

Amphotericin B acts by binding to ergosterol in the cell membrane of most fungi. After binding with ergosterol, it causes the formation of ion channels leading to loss of protons and monovalent cations, which results in depolarization and concentrationdependent cell killing. ${ }^{9,10}$ Oxidative damage, formation of free radicals, and increased membrane permeability and stimulation of phagocytic cells also assist in the clearing of fungal infections.

The half-life of amphotericin B is from 24 hours to 15 days. 
Table 1: Dose of various preparations of amphotericin B in neonates and children (7)

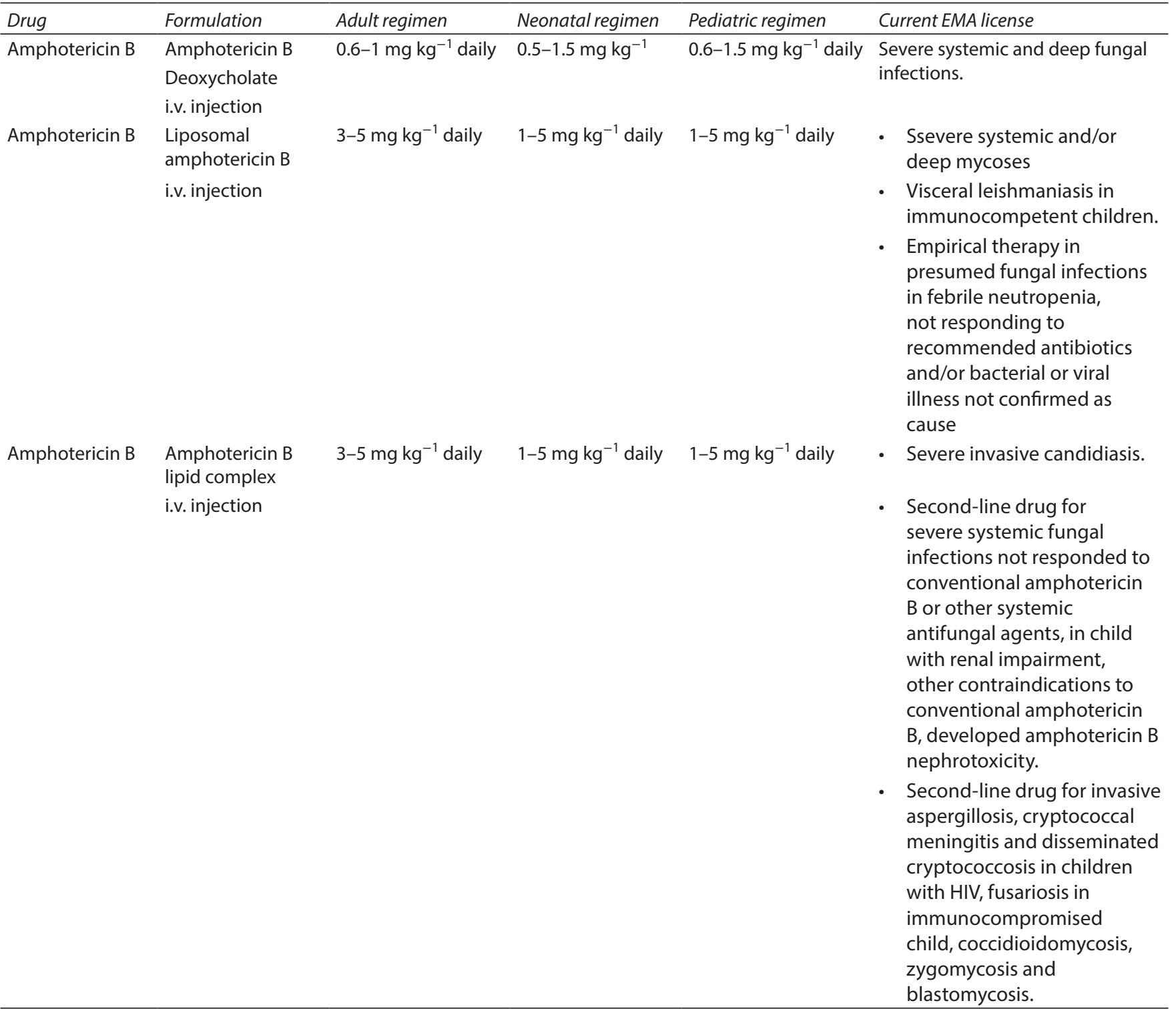

\section{Indications for Use of Amphotericin B in Children}

- Invasive candidiasis - It is effective against the majority of the Candida species, including Candida albicans, Candida krusei, Candida tropicalis, and Candida parapsilosis.

- In neonatal candidiasis, conventional amphotericin B is less toxic than in adults and well tolerated.

- Opportunistic fungal infections in immunocompromised children, including HIV $^{9,11}$

- Life-threatening fungal infections in both normal and immunocompromised hosts.

- Empiric treatment in a persistently febrile neutropenic host.

- Cerebral cryptococcosis along with flucytosine for induction therapy $^{12}$

- Based on their superior safety and efficacy, lipid formulations of amphotericin B have become the standard treatment for mucormycosis $^{13}$
- Severe cases of sporotrichosis: The treatment of choice for most cases is amphotericin B and subsequent itraconazole for maintenance therapy. ${ }^{14}$

- Coccidioidomycosis and paracoccidioidomycosis, especially in severe disease ${ }^{15}$

- The Infectious Diseases Society of America guidelines in 2007 recommended itraconazole for mild-to-moderate infection and amphotericin B for severe histoplasmosis. ${ }^{16}$ Amphotericin B is also being used a stepdown therapy for recurrent pulmonary histoplasmosis ${ }^{17}$ and for treating progressive disseminated histoplasmosis in people living with HIV. ${ }^{18}$

- Amphotericin B is usually recommended for severe blastomycosis in the lungs or infections that have spread to other parts of the body. ${ }^{19}$

- Aspergillosis for salvage therapy in cases not responding to voriconazole $^{10}$

- Visceral and cutaneous leishmaniasis ${ }^{9}$ 
Amphotericin B is the only agent in the polyene class of antifungals that is used for IFI. Amphotericin B deoxycholate, liposomal amphotericin B, amphotericin B lipid complex, and amphotericin $B$ colloidal dispersion have all been used to treat invasive fungal infections in neonates and children. ${ }^{9,10,20}$ Most of these studies suggest that amphotericin B use in this age-group should be guided by the following principles:

- All amphotericin B formulations penetrate the CNS (but not the (SF), which is important for the treatment of hematogenous Candida meningoencephalitis (HCME). However, most experts still recommended the use of amphotericin $B$ in this condition. ${ }^{21,22}$

- Amphotericin B does not achieve effective urinary concentrations and may therefore be ineffective for treatment of candiduria and renal fungal balls; however, low-dose amphotericin B may be useful as a second-line drug for Candida.

- Urinary tract infections in selected patients ${ }^{23}$ and amphotericin B bladder irrigation appears to be as effective as fluconazole for the condition, but it does not offer systemic antifungal therapy and should only be used for asymptomatic candiduria. ${ }^{24}$

- The pharmacokinetics of amphotericin B deoxycholate are exceedingly variable in neonates, and this may lead to unexpected treatment failure or toxicity. ${ }^{7}$

- Liposomal amphotericin B (L-AMB) is widely used in the treatment of invasive fungal infections in immunocompromised children; however, little is known about its safety and pharmacokinetics in this vulnerable patient population. ${ }^{25}$

- The lipid formulations of amphotericin B are increasingly used instead of amphotericin B deoxycholate, predominantly because of their more favorable safety profiles.

\section{Other Issues that are Being Considered}

It is extremely difficult to perform needle aspirations for culture in preterm low-birth-weight babies with fungal liver abscess. A report of Candida albicans hepatic abscess in a severely ill preterm neonate, being successfully treated with intralesional administration of L-AMPH-B (1 mg/mL, in isotonic water) is noted. ${ }^{26}$ More evidence of the safety of the procedure and its effectiveness in this condition needs to be available before recommending this line of treatment.

In case of central venous catheter and shunt-related candida infection, if these invasive devices are not immediately removed for any reason, micafungin should be preferred to other antifungal drugs such as amphotericin B or fluconazole, thanks to its ability to penetrate the biofilm that develops within the catheter. ${ }^{27}$

Administration $^{28}$

\section{IVInfusion}

Amphotericin B deoxycholate (Conventional formulation) - $50 \mathrm{mg}$ vial with $10 \mathrm{~mL}$ water for injections to give a solution of $5 \mathrm{mg}$ in $1 \mathrm{~mL}$. The displacement volume is negligible. This solution must then be further diluted to a concentration of 100 microgram in 1 $\mathrm{mL}$ with glucose $5 \%$. Concentrations up to 400 micrograms in $1 \mathrm{~mL}$ (unlicensed) may be used for fluid-restricted patients, provided administration is via a central line and by slow IV infusion over 4 to 6 hours. The duration of the infusion may be reduced to a minimum of 2 hours if the 4- to 6-hour infusion is tolerated. It is important to begin the infusion immediately after dilution and protect the solution from direct light. Amphotericin B deoxycholate is incompatible with sodium chloride solutions. Flush existing intravenous line with glucose $5 \%$ or use separate line; an in-line filter (pore size no less than 1 micron) may be used.

\section{Bladder Irrigation}

Reconstitute a 50-mg vial of conventional amphotericin with $10 \mathrm{~mL}$ water for injection to give a $5 \mathrm{mg}$ in $1 \mathrm{~mL}$ solution. Further, dilute $2 \mathrm{~mL}$ of this solution to $100 \mathrm{~mL}$ with water for irrigation to produce a $100 \mu \mathrm{g}$ in $1 \mathrm{~mL}$ solution or 1 to $100 \mathrm{~mL}$ to produce a $50 \mu \mathrm{g}$ in $1-\mathrm{mL}$ solution for instillation into the bladder.

\section{Liposomal Amphotericin B}

It is available as $50-\mathrm{mg}$ powder for injection. The maximum permissible concentration (MPC), the maximum dose of a drug which can be diluted per $\mathrm{mL}$ of the diluent, for liposomal amphotericin B is $2 \mathrm{mg} / \mathrm{mL}$ for infants (small children: 0.2 to $0.5 \mathrm{mg} / \mathrm{mL}$ ). Reconstitute $50-\mathrm{mg}$ vial with $12 \mathrm{~mL}$ water for injections to give a solution of $4 \mathrm{mg}$ in $1 \mathrm{~mL}$. This solution must then be filtered, using the 5-micron filter provided into the diluent solution of glucose $5 \%$ only. Dilute to a concentration between 200 microgram and $2 \mathrm{mg}$ in $1 \mathrm{~mL}$. Administer as a slow IV infusion over 1 hour. The infusion may be administered over 30 minutes if well tolerated or over more than 1 hour if acute infusion reactions occur.

\section{Contraindications ${ }^{28}$}

Lipid formulations, particularly liposomal amphotericin, are less likely to cause severe side effects-still it is advisable to monitor for hypersensitivity to amphotericin or any constituents of the formulations. Renal impairment may occur, and discontinuation or reduction should be considered if urea or serum creatinine exceeds twice the upper limit of normal. Monitor serum potassium, magnesium, and phosphate as low serum levels may occur. Monitor LFTs and consider discontinuation if seriously deranged. Avoid concurrent nephrotoxic drugs, antineoplastic agents if possible, and also corticosteroids unless necessary to control drug reactions.

\section{Side Effects ${ }^{28}$}

\section{Conventional Formulation}

Acute infusion reactions are the most frequent adverse reactions such as fever, shaking, chills, nausea, vomiting, headache, dyspnea, tachypnea, gastrointestinal effects, muscle and joint pains, and pain at site of injection.

These reactions are most severe and occur most frequently with initial doses and often lessen with subsequent doses. Febrile reactions may be managed by IV administration of hydrocortisone but keep dose and duration to a minimum. Nephrotoxicity, to some degree, occurs in $85 \%$ of children. Hypokalemia, hypomagnesemia, renal tubular acidosis, nephrocalcinosis, and hyposthenuria may occur. Hyperkalemia and arrhythmias can result if administered too rapidly. Decreased creatinine clearance, reduction in glomerular filtration rate, and decreased renal blood flow are seen. Renal function generally improves within a few months, although some degree of permanent impairment may occur. Patients with higher serum low-density lipoprotein (LDL) concentrations may be more susceptible to renal toxicity. Less common renal toxicity reactions are anuria, oliguria, hematuria, urinary incontinence, and acute renal failure. Acute reactions, hypotension, fever, chills may occur within 2 hours. Amphotericin B Lipid complex may cause fever, rash, headache, hematological abnormalities, hepatotoxicity, and gastrointestinal abnormalities. 


\section{Conclusion}

Pediatricians, who prescribe amphotericin B, should be familiar with its administration and side effects. Three-fourth of children receiving amphotericin $B$ could develop either infusion-related or renal toxicity. Administration of acetaminophen, diphenhydramine, and/or hydrocortisone prior to infusion may reduce infusionrelated side effects. Following infusion, the renal function requires monitoring.

\section{References}

1. Groll AH, Piscitelli SC, Walsh TJ. Clinical pharmacology of systemic antifungal agents: a comprehensive review of agents in clinical use, current investigational compounds, and putative targets for antifungal drug development. Adv Pharmacol 1998;44:343-500. DOI: 10.1016/s1054-3589(08)60129-5.

2. Zaoutis $T E$, Coffin $\mathrm{SE}, \mathrm{Chu} \mathrm{JH}$, et al. Risk factors for mortality in children with candidemia. Pediatr Infect Dis J 2005;24(8):736-739. DOI: 10.1097/01.inf.0000172938.76561.8e.

3. Mesini A, Bandettini R, Caviglia l, et al. Candida infections in paediatrics: results from a prospective single-centre study in a tertiary care children's hospital. Mycoses 2016;60(2):118-123. DOI: 10.1111/ myc.12570.

4. Playford EG, Lipman J, Sorrell TC. Prophylaxis, empirical and preemptive treatment of invasive candidiasis. Curr Opin Crit Care 2010;16(5):470-474. DOI: 10.1097/MCC.0b013e32833e10e8.

5. Brissaud O, Guichoux J, Harambat J, et al. Invasive fungal disease in PICU: epidemiology and risk factors. Ann Intensive Care 2012;2(1):6. DOI: 10.1186/2110-5820-2-6.

6. Kontoyiannis DP, Marr KA, Park BJ, et al. Prospective surveillance for invasive fungal infections in hematopoietic stem cell transplant recipients, 2001-2006: overview of the transplant-associated infection surveillance network (TRANSNET) database. Clin Infect Dis 2010;50(8):1091-1100. DOI: 10.1086/651263.

7. Lestner JM, Smith PB, Cohen-Wolkowiez M, et al. Antifungal agents and therapy for infants and children with invasive fungal infections: a pharmacological perspective. Br J Clin Pharmacol 2013;75(6):13811395. DOI: 10.1111/bcp.12025.

8. Ghannoum MA, Rice LB. Antifungal agents: mode of action, mechanisms of resistance, and correlation of these mechanisms with bacterial resistance. Clin Microbiol Rev 1999;12(4):501-517. DOI: 10.1128/CMR.12.4.501.

9. Al Balushi A, Khamis F, Klaassen $\mathrm{CHW}$, et al. Double infection with leishmania tropica and L. major in an HIV patient controlled with high doses of amphotericin B. Open Forum Infect Dis. 2018;5(12):ofy323. DOI: 10.1093/ofid/ofy323.

10. Rybak JM, Fortwendel JR, Rogers PD. Emerging threat of triazole-resistant aspergillus fumigatus. J Antimicrob Chemother 2019;74(4):835-842. DOI: 10.1093/jac/dky517.

11. Guidelines for the Prevention and Treatment of Opportunistic Infections Among HIV-Exposed and HIV-Infected Children. https:// aidsinfo.nih.gov/contentfiles/lvguidelines/glchunk/glchunk_398. pdf. Accessed on May 16, 2020.

12. Schwarz P, Dromer F, Lortholary O, et al. Efficacy of amphotericin $B$ in combination with flucytosine against flucytosine-susceptible or flucytosine-resistant isolates of Cryptococcus neoformans during disseminated murine cryptococcosis. Antimicrob Agents Chemother 2006;50(1):113-120. DOI: 10.1128/AAC.50.1.113-120.2006.

13. Spellberg B, Ibrahim AS. Recent advances in the treatment of mucormycosis. Curr Infect Dis Rep 2010;12(6):423-429. DOI: 10.1007/ s11908-010-0129-9.

14. Bonifaz A, Tirado-Sánchez A. Cutaneous disseminated and extracutaneous sporotrichosis: current status of a complex disease. J Fungi (Basel) 2017;3(1):6. DOI: 10.3390/jof3010006.

15. Salzer HJF, Burchard G, Cornely OA, et al. Diagnosis and management of systemic endemic mycoses Causing pulmonary disease. Respiration 2018;96(3):283-301. DOI: 10.1159/000489501.

16. Wheat LJ, Freifeld AG, Kleiman MB, et al. Infectious diseases society of America. clinical practice guidelines for the management of patients with histoplasmosis: 2007 update by the infectious diseases society of America. Clin Infect Dis 2007;45(7):807-825. DOI: 10.1086/521259.

17. Lewis PO, Khan I, Patel P. Successful stepdown treatment of pulmonary histoplasmosis with thrice-weekly liposomal amphotericin B in a hospital-associated, outpatient infusion centre: a case report. J Clin Pharm Ther 2018;43(2):269-272. DOI: 10.1111/jcpt.12609.

18. Murray M, Hine P. Treating progressive disseminated histoplasmosis in people living with HIV. Cochrane Database Syst Rew 2020;4:CD013594. DOI: 10.1002/14651858.CD013594.

19. Pelly L, Juaid A, Fanella S. Severe blastomycosis in infants. Pediatr Infect Dis J 2014;33(11):1189-1191. DOI: 10.1097/INF.0000000000000400.

20. Linder N, Klinger G, Shalit I, et al. Treatment of candidaemia in premature infants: comparison of three amphotericin $B$ preparations. J Antimicrob Chemother 2003;52(4):663-667. DOI: 10.1093/jac/ dkg419.

21. Walsh TJ, Katragkou A, Chen T, et al. Invasive candidiasis in infants and children: recent advances in epidemiology, diagnosis, and treatment. J Fungi 2019;5(1):pii: E11. DOI: 10.3390/jof5010011.

22. Greenberg RG, Benjamin DKJr. Neonatal candidiasis: diagnosis, prevention, and treatment. J Infect 2014;69(Suppl 1):S19-22. DOI: 10.1016/j.jinf.2014.07.012.

23. Fisher JF, Sobel JD, Kauffman CA, et al. Candida urinary tract infections-treatment. Clin Infect Dis 2011;52(suppl_6):S457-S466. DOI: $10.1093 / \mathrm{cid} / \mathrm{cir} 112$.

24. Tuon FF, Amato VS, Filho SRP. Bladder irrigation with amphotericin $B$ tract and fungal urinary infection-systematic review with meta-analysis. Int J Infect Dis 2009;13(6):701-706. DOI: 10.1016/j. ijid.2008.10.012.

25. Seibel NL, Shad AT, Bekersky I, et al. Safety, Tolerability, and pharmacokinetics of liposomal amphotericin $B$ in immunocompromised pediatric patients. Antimicrob Agents Chemother 2016;61(2):e01477-16. DOI: 10.1128/AAC. 01477-16.

26. Auriti C, Ronchetti MP, Bersani I, et al. Intrahepatic administration of liposomal amphotericin B (ambisome) for the management of a liver abscess from Candida albicans in a preterm infant. Antimicrob Agents Chemother 2018;62(12):e01239-18. DOI: 10.1128/AAC.01239-18.

27. Swaminathan S, Kamat S, Pinto NA. Echinocandins: their role in the management of Candida biofilms. Indian J Med Microbiol 2018;36(1):87-92. DOI: 10.4103/ijmm.IJMM_17_400.

28. Amphotericin, In: IAP Drug Formulary Unni JC, Nair MKC, Menon PSN 5th ed., Cochin: Pixel Studio; 2019. pp. 279-280. 